\title{
Deep Brain Stimulation In Iran: Where It Began and Where It Goes
}

\author{
Feryal Momenilandi (ii) ${ }^{1, *}$ and Sepand Tehrani Fateh (iD) ${ }^{2,1}$ \\ ${ }^{1}$ Functional Neurosurgery Research Center, Shohada Tajrish Neurosurgical Center of Excellence, Shahid Beheshti University of Medical Sciences, Tehran, Iran \\ ${ }^{2}$ School of Medicine, Shahid Beheshti University of Medical Sciences, Tehran, Iran \\ "Corresponding author: Functional Neurosurgery Research Center, Shohada Tajrish Neurosurgical Center of Excellence, Shahid Beheshti University of Medical Sciences, \\ Tehran, Iran. Email: feryal.ml.71@gmail.com
}

Received 2021 September 06; Revised 2021 September 22; Accepted 2021 September 22.

Keywords: Tourette, Dystonia, Parkinson's Disease, History, DBS

\section{Dear Editor,}

Deep brain stimulation (DBS) is a surgical implementation of electrodes into specific brain regions to recover the physiological activities of neural circuits or suppress pathological impulses via electrical stimulation. This procedure has been used in a variety of neurological and psychiatric disorders (1).

The early applications of DBS in the clinic were dedicated to analgesic purposes during the 1970s; however, not very satisfying results were obtained (2). About two decades later, in the late 1980s, DBS found its way into the field of involuntary movement disorders (2). In the late 20 th century and early 21 st century, the FDA approved DBS for some movement disorders (2). Right after, the idea of treating complicated diseases (e.g., obsessive-compulsive disease, obesity, Tourette, and epilepsy) with this procedure was ignited (2). As agreed upon, 2002 stands as the year in which DBS was granted conditional approval in its applicability in the treatment of $\mathrm{PD}(2)$.

Early attempts of DBS in Iran possibly stem from the treatment of PD patients by Haghi-Ashtiani et al. in 2004 (3). By reporting approximately successful results concerning the dosage of medications and improvement of motor symptoms, they progressed to a new chapter in treating those affected by PD's refractory symptoms. During recent years, DBS has been used for other varieties of neuropsychiatric disorders in Iran, such as dystonia $(4,5)$, Tourette syndrome (6), and Holmes tremor (7). However, DBS is still in the embryonic stages of its life in Iran since this procedure is mostly limited to the hospitals affiliated with medical universities (e.g., Shahid Beheshti, Iran, and Shiraz Universities of Medical Sciences). However, the centralization of facilities and experts in some medical universities in large cities is a reason for limited access to this treatment. On the other hand, many patients are unwilling to undergo such a procedure due to its invasiveness and their dependency on a specialist for post-care surgery and adjustment of the device. Altogether, the limited number of patients would lead to much less experience in this field, lack of sufficient reports of adverse effects, lack of the popularity of the method among physicians and patients, and lack of priority for policymakers to dedicate relevant medical facilities or proper insurance services.

Several solutions from multiple aspects should be implemented to address these issues. Clinical studies on DBS application in Iranian patients are mostly limited to single case reports or case series, and more case-control studies and trials are required. These studies should not be limited to the outcome of this procedure, but its practicality regarding the lifestyle and cultural considerations of Iranian patients have to be investigated. In this case, the lack of trust among both patients and physicians may be compensated. Creating educational materials for patients and physicians would be another possible solution for proper decision-making. The significance of this medical procedure for policymakers should also be highlighted to decentralize proper medical facilities for DBS to be undertaken. Moreover, we strongly encourage authorities and colleges to establish a national database of DBS surgeries and their outcomes to obtain a clear perspective and cooperative atmosphere on this issue.

\section{Footnotes}

Authors' Contribution: Study concept and design, FM and STF; Acquisition of data, FM and STF; Analysis and interpretation of data, FM and STF; Drafting of the manuscript, FM and STF; Critical revision of the manuscript for important intellectual content, FM; Statistical analysis, STF; Administrative, technical, and material support, FM; Study 


\section{supervision, FM.}

Conflict of Interests: The authors do not report any conflicts of interest.

Funding/Support: It was not declared by the authors.

\section{References}

1. Krauss JK, Lipsman N, Aziz T, Boutet A, Brown P, Chang JW, et al. Technology of deep brain stimulation: Current status and future directions. Nat Rev Neurol.2021;17(2):75-87. doi:10.1038/s41582-020-00426-z. [PubMed: 33244188]. [PubMed Central: PMC7116699].

2. Coffey RJ. Deep brain stimulation devices: A brief technical history and review. Artif Organs. 2009;33(3):208-20. doi: 10.1111/j.15251594.2008.00620.x. [PubMed: 18684199].

3. Haghi-Ashtiani B, Shahidi G, Rohani M, Jalili M, Sina F. Early outcome of subthalamic nucleus deep brain stimulation (STN-DBS) in advanced parkinson disease in first trial of Iranian patients. Med J Islam Repub Iran. 2009;23(3):122-7.
4. Miri S, Ghoreyshi E, Shahidi GA, Parvaresh M, Rohani M, Saffari M Deep brain stimulation of globus pallidus internus for DYT1 positive primary generalized dystonia. Med J Islam Repub Iran. 2014;28:18 [PubMed: 25250263]. [PubMed Central: PMC4153526].

5. Ramezani Ghamsari M, Ghourchian S, Emamikhah M, Safdarian M, Shahidi G, Parvaresh M, et al. Long term follow-up results of deep brain stimulation of the Globus pallidus interna in pediatric patients with DYT1-positive dystonia. Clin Neurol Neurosurg. 2021;201:106449. doi: 10.1016/j.clineuro.2020.106449. [PubMed: 33395620].

6. Azimi A, Parvaresh M, Shahidi G, Habibi A, Rohani S, Safdarian M, et al Anteromedial GPi deep brain stimulation in Tourette syndrome: The first case series from Iran. Clin Neurol Neurosurg. 2018;172:116-9. doi: 10.1016/j.clineuro.2018.06.045. [PubMed: 29990958].

7. Razmkon A, Yousefi O, Vaidyanathan J. Using preimplanted deep brain stimulation electrodes for rescue thalamotomy in a case of holmes tremor: A case report and review of the literature. Stereotact Funct Neurosurg. 2020;98(2):136-41. doi: 10.1159/000506083. [PubMed: 32209790]. 